\title{
EFEITOS DO AGROSTEMIN EM PLANTAS DE SOJA (Glicine $\max ($ (L.) MERRILL CV. IAC-8), ATRAVÉS DOS PARÂMETROS FISIOLÓGICOS: RAZÃO DE ÁREA FOLIAR, TAXA ASSIMILATÓRIA LÍQUIDA E TAXA DE CRESCIMENTO RELATIVO'.
}

\author{
A.A. H. FERNANDES ${ }^{2}$; J.D. RODRIGUES ${ }^{2} ;$ P.R.C. CASTRO $;$; S.Z. DE PINHO ${ }^{4}$ \\ 2- Depto. de Botânica - IB/UNESP, CEP: 18618-000-Botucatu, SP. \\ 3- Depto. de Botânica - ESALQUSP, C.P. 9, CEP: 13418-900 - Piracicaba S.P. \\ 4- Depto. de Bioestatistica IB/UNESP, CEP: 18618-000 - Botucatu, S.P.
}

RESUMO: O presente estudo teve por finalidade avaliar o efeito de um estimulante vegetal, Agrostemin, no desenvolvimento de plantas de soja (Glycine max (L.) Merrill cv. IAC-8), através de alguns parâmetros que compōem a análise de crescimento. $O$ experimento fol conduzido em casa de vegetaçāo. Foram estudados seis tratamentos, correspondentes às seguintes dosagens, épocas e formas de aplicação: testemunha ( $\left.T_{1}\right) ; 0,125 \mathrm{~g}$ de Agrostemin $100 \mathrm{~g}$ sementes $\left(\mathrm{T}_{2}\right) ; 0,125 \mathrm{~g}$ de Agrostemin $/ 100 \mathrm{~g}$ sementes e pulverização foliar à $333 \mathrm{ppm}\left(\mathrm{T}_{\mathfrak{y}}\right) ; 0,125 \mathrm{~g}$ de Agrostemin $/ 100 \mathrm{~g}$ sementes e pulverização foliar à $500 \mathrm{ppm}\left(T_{4}\right)$; pulverização foliar à $333 \mathrm{ppm}\left(T_{3}\right)$ e pulverização foliarà $500 \mathrm{ppm}\left(T_{\text {q }}\right)$. Realizaram-se três épocas de coleta, à intervalos de 14 dias. $O$ experimento fol em delineamento inteiramente casualizado. Os parâmetros estudados foram: razão de área foliar (RAF), taxa assimilatória liquida (TAL) e taxa de crescimento relativo (TCR). Dentre os resultados obtidos, conclưu-se que à medida que aumentou-se a concentração, diminuiram-se os parâmetros estudados, no intervalo 71-85.

Descritores: Glycine max L., Agrostemin, análise de crescimento

\section{EFFECTS OF AGROSTEMMIN ON SOYBEAN PLANTS [Glicine max (L.) MERRIL cv. IAC -8] THROUGH PHYSIOLOGICAL PARAMETERS: NET ASSIMILATION, FOLIAR AREA RATIO AND RELATIVE GROWTH RATE.}

\begin{abstract}
The objetive of this work was to evaluate the effect of Agrostemmin on the development of soybean (Glycine max (L.) Merrill cv. IAC-8) plants through physiological parameters related to plant growth. The experiment was carried out in a greenhouse.Six treatments corresponding to the following dosages, time and application ways were studied: control $\left(T_{1}\right) ; 0,125 \mathrm{~g}$ Agrostemmin/100g seed $\left(T_{2}\right) ; 0,125 \mathrm{~g}$ Agrostemmin/100g seed and foliar spraying at $333 \mathrm{ppm}\left(T_{3}\right)$; $0,125 \mathrm{~g}$ Agrostemmin/100g seed and foliar spraying at $500 \mathrm{ppm}\left(\mathrm{T}_{\text {}}\right.$ ); foliar spraying at $333 \mathrm{ppm}\left(\mathrm{T}_{2}\right.$ ) and foliar spaying at $500 \mathrm{ppm}(\mathrm{T})$. Three samplings were made at 14 day intervals. The experimental layout was completely randomized. The parameters studied were: foliar area ratio (FAR), net assimilation rate (NAR) and relative growth rate (RGR). It could be concluded that in proportion to increased rates there was a decrease in the parameters, in intervals 71-85.
\end{abstract}

Key words: soybean, Agrostemmin, growth analysis

\section{INTRODUÇĀO}

A melhoria na produção de soja, através da utilização de reguladores de crescimento, de acordo com Smith (1978), citado por CASTRO \& MORAES (1981), tem recebido nos últimos anos grande ênfase nas pesquisas realizadas pelas indústrias, universidades e agências governamentais. Entretanto, o desenvolvimento de compostos potencialmente ativos, que poderiam aumentar significativamente a produção, tem sido limitado por inúmeras variáveis. Além das limitações fisiológicas, tais como, a fotossíntese e a eficiência da fixação de nitrogênio, os fatores ambientais também interferem na produção de sementes. Assim, a habilidade de um regulador vegetal em favorecer o desenvolvimento ou estimular a produção, pode não ser aparente em função de fatores ambientais limitantes.

STANKOVIC \& RAJKOVIC (1984), registraram que a descoberta do Agrostemin foi feita por Danica Gajic, aproximadamente há 30 anos, através de investigações sistemáticas sobre as interrelações alelopáticas, entre plantas de trigo e Agrostemma githago L.

Dessa forma, vários trabalhos são encontrados apresentando os efeitos de Agrostemin, como o

1- Parte da dissertação de Mestrado, apresentado pelo lo Autor, ao IB/UNESP, Botucatu - SP, financiada pela FAPESP.

Sci. agric., Piracicaba, 52(2):339-345, mai./ago. 1995 
de GAJC \& VRBASKI (1972), ao relatarem que o estimulante vegetal aumentou o crescimento, tanto da raiz, como da parte aérea de trigo.

RADFORD (1967), apresentou uma série de fórmulas para determinar alguns parâmetros fisiológicos, discutindo as condições necessárias para o seu uso correto. Além disto, propôs um método alternativo para a análise de crescimento, envolvendo uma descrição matemática que se baseia na massa da matéria seca e na área foliar em função do tempo, acompanhado de cálculo das estimativas instantâneas dos diferentes parâmetros da análise de crescimento.

Por sua vez, CASTRO (1981), estudando os efeitos da pulverização de Agrostemin na préfloração em soja "Davis", verificou que a substância promoveu aumento na área foliar e na taxa assimilatória líquida, revelando que o ganho em produtos da fotossintese por unidade de área foliar foi maior nestas plantas, mostrando o efeito estimulatório desse biorregulador. $\mathrm{O}$ mesmo autor, cita que a taxa de crescimento relativo mostrou-se mais elevada nas plantas tratadas, indicando que o estimulante vegetal aumentou a produção de matéria seca por unidade de área foliar.

Considerando os fatores mencionados, os autores se propuseram a desenvolver um estudo sobre alguns parâmetros fisiologicos de plantas de soja (Glycine max (L.) Merrill cv. IAC-8), quando submetidas a diferentes tratamentos com Agrostemin.

\section{MATERIAL E MÉTODOS}

O experimento foi conduzido em condições de casa de vegetação, no Departamento de Botânica do Instituto de Biociências, Campus de Botucatu, UNESP.

Para a execução do trabalho, utilizou-se o produto comercial Agrostemin, tendo como principal composição química os seguintes aminoácidos: alantoina, triptofano, ácido fólico, ácido alantóico, ácido glutâmico e adenina. Com essa substância, realizaram-se seis tratamentos, com três repetições, correspondentes às seguintes dosagens, épocas e formas de aplicação:

$T_{1}:$ Testemunha;

$\mathrm{T}_{2}: 0,125 \mathrm{~g}$ Agrostemin $/ 100 \mathrm{~g}$ de sementes;

$\mathrm{T}_{3}: 0,125 \mathrm{~g}$ Agrostemin $100 \mathrm{~g}$ de sementes e pulverização foliar à $333 \mathrm{ppm}$.

$\mathrm{T}_{4}: 0,125 \mathrm{~g}$ Agrostemin $/ 100 \mathrm{~g}$ de sementes e pulverização foliar à $500 \mathrm{ppm}$

$\mathrm{T}_{s}$ : pulverização foliar à $333 \mathrm{ppm}$ e

$T_{6}$ : pulverização foliar à $5000 \mathrm{ppm}$.
De acordo com as instruções contidas na embalagem do produto comercial, sua aplicação foi feita diretamente na semente, a seco, previamente à semeadura. As pulverizações foliares foram feitas na pré-floração, ou seja, 43 dias após o plantıo Foram efetuadas três coletas de planta, a intervalos de 14 dias entre si, tendo início aos 14 dias após as pulverizações foliares, ou seja, as coletas I, II e III foram efetuadas aos 57,71 e 85 dias após o plantio, respectivamente.

A partir da média de duas plantas analisadas para cada repetição, obtiveram-se as medidas biométricas, ou seja, massa da matéria seca para cada órgão separadamente e a área foliar, a qual foi medida através do medidor de superficie laminar, modelo "MSL-80".

Através das medidas acima citadas, calcularam-se os seguintes parâmetros fisiológicos que compõem a análise de crescimento

Razão de área foliar (RAF), representa a relação entre a área foliar total (AF) e a massa da matéria seca total (MST), resultado desta fotossíntese, expressa em decímetros quadrados por grama (BENINCASA ,1988), seguindo a relação.

$$
\mathrm{RAF}=\frac{\mathrm{AF}}{\mathrm{MST}}
$$

Taxa assimilatória líquida (TAL), reflete a eficiência do sistema assimilador que está envolvido na produção de matéria seca, estimando, dessa forma, a fotossintese liquida (RADFORD, 1967). definida pela expressão.

\section{$T A L=\frac{M S T n-M T n-1}{A F n-A F n-1} \times \underline{\ln A F n-\ln A F n-1}$ $n \rightarrow n-1 \quad A F n-A F n-1 \quad$ tn - tn-1}

onde:

$\mathrm{n}=$ número de coletas;

$t=$ tempo entre coletas, em dias;

MST = massa da matéria seca total;

$\mathrm{AF}=$ área foliar.

Sendo expressa $\mathrm{em} \mathrm{g} / \mathrm{dm}^{2}$ xdia

-Taxa de crescimento relativo (TCR), reflete o aumento da matéria seca em gramas, de uma planta ou de qualquer órgão, num dado intervalo de tempo, em função do tamanho inicial, ou seja, de material pré-existente (BENINCASA, 1988), sendo definida pela expressão:

$\underset{n \rightarrow n-1}{\mathrm{TCR}}=\underset{n \rightarrow n-1}{\mathrm{TAL}} \times \frac{\text { RAF } n+R A F n-1}{2}$

onde: 
$\mathbf{n}=$ número de coletas;

$\mathrm{TAL}=$ taxa assimilatória líquida;

$\mathrm{RAF}=$ razão de área foliar,

Sendo expressa em $\mathbf{g} / \mathbf{g x d i a}$.

$O$ delineamento experimental utilizado, foi inteiramente casualizado. Não foi realizada análise de variância, uma vez que não se pode afirmar que essas variáveis, por serem calculadas, obedeçam às pressuposições básicas dessa análise (BANZATTO \& KRONKA, 1989).

\section{RESULTADOS E DISCUSSÃO}

\section{Razão de área foliar}

A TABELA 1, mostra os resultados obtidos para a razão de área foliar, considerando-se os tratamentos T1, T5 e T6. As plantas ao receberem Agrostemin, via foliar, à $500 \mathrm{ppm}\left(\mathrm{T}_{6}\right)$, demonstraram maiores valores para a razão de área foliar, em relação aos demais tratamentos, os quais praticamente não diferiram entre si, aos 57 e 71 dias pós-plantio. Porém, este parâmetro fisiológico decresceu consideravelmente nas plantas que receberam Agrostemin, comparativamente à testemunha, aos 85 dias pós-plantio.Estes resultados, estão de acordo com os achados por CASTRO (1981), o qual ao aplicar Agrostemin via foliar em soja, verificou que a razão de área foliar foi menor no final do ciclo vegetativo, comparativamente à testemunha (Figura 1).

Como a razão de área foliar representa a proporção de material fotossintetizante, em relação a massa da matéria seca total da planta (MAGALHÃES, 1986), pode ser sugerido que os tratamentos T5 e T6, aos 85 dias após o plantio, apresentaram menor quantidade de material fotossintetizante em relação a massa da matéria seca total.

Considerando-se a aplicação de Agrostemin, tanto nas sementes como nas folhas, nota-se através da TABELA 1A e Figura 1A, que as plantas pertencentes aos tratamentos $T_{3}$ e $T_{4}$, apresentaram valores inferiores, comparativamente à testemunha, aos 71 dias pós-plantio. A partir deste périodo, trona-se nítido o declínio neste parâmetro, quando as plantas oriundas de sementes tratadas, receberam o bioestimulante através de pulverizações foliares $\left(\mathrm{T}_{3}\right.$ e $\left.T_{4}\right), e m$ relação àquelas tratadas somente através das sementes. Desta forma, pode-se inferir que, provavelmente, o Agrostemin aplicado em dois estádios, via semente e via foliar,não contribuiu para aumentar efetivamente a área foliar útil para a fotossintese o que, consequentemente, iria refletirse em últirna análise, em ganho na massa da matéria seca total.

TABELA 1: Valores médios obtidos para a razão de área foliar, considerando-se os tratamentos $T_{1}, T_{5}$ e $\mathrm{T}_{6}$, aos 57,71 e 85 dias pós-plantio, expressos $\mathrm{em} \mathrm{dm}^{2} / \mathrm{g}$.

\begin{tabular}{lccc}
\hline \hline Tratamentos & \multicolumn{3}{c}{ dias pós-plantio } \\
\cline { 2 - 4 } & 57 & 71 & 85 \\
$\mathrm{~T}_{1}$ & 0,543 & 0,509 & 0,506 \\
$\mathrm{~T}_{5}$ & 0,535 & 0,496 & 0,455 \\
$\mathrm{~T}_{6}$ & 0,577 & 0,570 & 0,412 \\
\hline
\end{tabular}

TABELA 1A: Valores médios obtidos para a razáo de área foliar, considerando-se os tratamentos $T_{2}, T_{3}$ e $T_{4}$, aos 57,71 e 85 dias pós-plantio, expressos $\mathrm{em} \mathrm{dm}^{2} / \mathrm{g}$.

\begin{tabular}{lccc}
\hline \hline Tratamentos & \multicolumn{3}{c}{ dias pós-plantio } \\
\cline { 2 - 4 } & 57 & 71 & 85 \\
\cline { 2 - 4 } $\mathrm{T}_{2}$ & 0,501 & 0,701 & 0,482 \\
$\mathrm{~T}_{3}$ & 0,442 & 0,567 & 0,383 \\
$\mathrm{~T}_{4}$ & 0,516 & 0,525 & 0,398
\end{tabular}




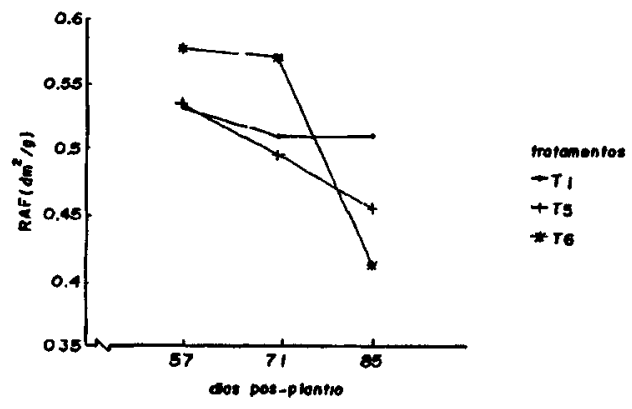

Figura 1: Valores médios obtidos para razão de área foliar, considerando-se as plantas que receberam Agrostemin, via foliar.

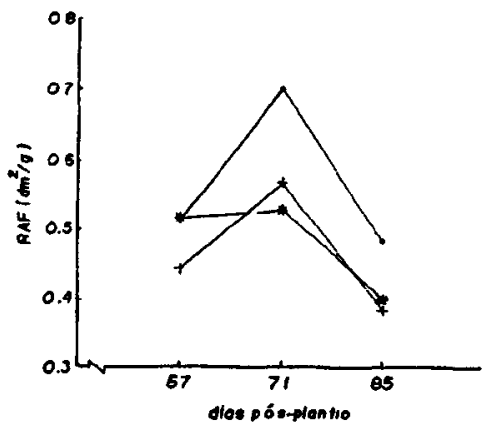

irotomontos

$-12$

$+73$

$+14$

Figura 1A: Valores médios obtidos para razão de área foliar, considerando-se as plantas que receberam Agrostemin, tanto por via foliar como por semente.
Taxa assimilatória líquida

$\mathrm{Na}$ TABELA 2, encontram-se os resultados médios obtidos para a taxa assimilatória líqui$\mathrm{da}$, considerando-se as plantas que receberam Agrostemin somente através das pulverizações foliares.

Tomando-se como referência o comportamento das plantas pela Figura 2, verifica-se que ao receberem Agrostemin via foliar, apresentaram redução neste parâmetro, em relação à testemunha, no intervalo entre 57-71. Entretanto, no intervalo seguinte (71-85), a taxa assimilatória líquida reduziuse drasticamente nas plantas representadas pelo tratamento $T_{1}$, atingido valores próximos aos obtidos para as plantas tratadas $\left(T_{5}\right.$ e $\left.T_{6}\right)$. CASTRO et al. (1987), não evidenciaram qualquer variação na taxa assimilatória líquida, em plantas de milho submetidas à aplicação de Agrostemin, via foliar. Por outro lado, CASTRO (1981), estudando o efeito de Agrostemin em soja, observou aumento da taxa assimilatória liquida, revelando assim maiores ganhos em produtos da fotossíntese por unidade de área foliar.

Considerando-se as plantas que receberam Agrostemin, tanto nas sementes como nas folhas (TABELA 2A e Figura 2A), verifica-se aumento considerável na taxa assimilatória líquida quando empregou-se $333 \mathrm{ppm}$ do bioestimulante, via foliar, sobre plantas originárias de sementes tratadas $\left(\mathrm{T}_{3}\right)$ e redução quando a concentração do estimulante ve-

TABELA 2: Valores médios obtidos para a taxa assimilatória líquida, considerando-se os tratamentos $T_{1}, T_{5} e$ $T_{6}$, nos intervalos 57-71 e 71-84 dias pós-plantio, expressos em g/dm² $x$ dia.

\begin{tabular}{|c|c|c|}
\hline \multirow[t]{2}{*}{ Tratamentos } & \multicolumn{2}{|c|}{ Intervalos } \\
\hline & $57-71$ & $71-84$ \\
\hline$T_{1}$ & 0,041 & 0,001 \\
\hline$T_{5}^{1}$ & 0,012 & 0,016 \\
\hline $\mathrm{T}_{6}^{5}$ & 0,024 & 0,008 \\
\hline
\end{tabular}

TABELA 2A: Valores médios obtidos para a taxa assimilatória líquida, considerando-se os tratamentos $T_{2}, T_{3}$ e $T_{4}$, nos intervalos 57-71 e 71-84 dias pós-plantio, expressos em $\mathrm{g} / \mathrm{dm}^{2} \mathrm{xdia}$.

\begin{tabular}{lll}
\hline \hline Tratamentos & \multicolumn{2}{c}{ Intervalos } \\
\cline { 2 - 3 } & $57-71$ & $71-84$ \\
$\mathrm{~T}_{2}$ & 0,006 & 0,030 \\
$\mathrm{~T}_{3}$ & 0,015 & 0,019 \\
$\mathrm{~T}_{4}$ & 0,007 & 0,016 \\
\hline \hline
\end{tabular}


getal elevou-se $\left(\mathrm{T}_{4}\right)$, no intervalo $57-71$, em relação ao tratamento $\mathrm{T}_{2}$. Contudo, no intervalo seguinte (7185), a taxa assimilatória liquida aumentou consideravelmente nas plantas que receberam Agrostemin somente através das sementes, comparativamente aos demais tratamentos. Dessa forma, pode-se inferir que o Agrostemin aplicado em dois estádios, contribuiu para diminuir a taxa assimilatória líquida em plantas de soja, sendo esta redução maior quando empregou-se a concentração de $500 \mathrm{ppm}$.

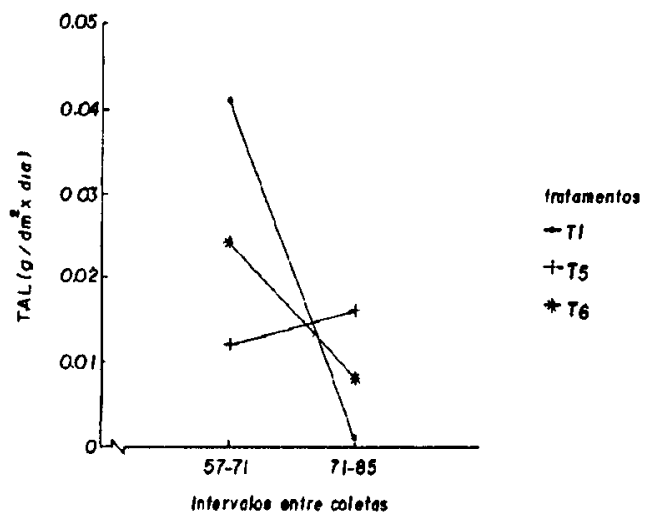

Figura 2: Valores médios obtidos para taxa assimilatória liquida, considerando-se as plantas que receberam Agrostemin, via foliar

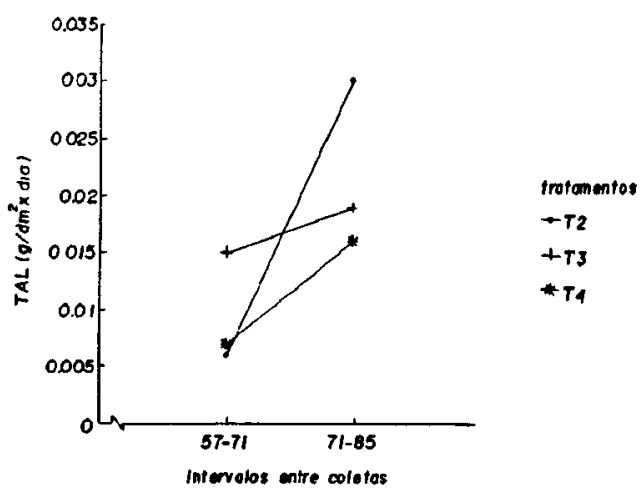

Figura 2A: Valores médios obtidos para taxa assmilatória líquida, considerando-se as plantas que receberam Agrostemin, tanto por via foliar como por semente.

\section{Taxa de crescimento relativo}

Através dos resultados obtidos, expressos na TABELA 3 e Figura 3, pode-se observar o comportamento das plantas submetidas à aplicação de Agrostemin, via foliar. No intervalo (57-71), as plan- tas pertencentes ao tratamento $T_{5}$ apresentaram valores inferiores às demais plantas. Comportamento totalmente oposto, pode ser observado no intervalo seguinte (71-85), onde as plantas pertencentes ao tratamento $T_{\text {s }}$ apresentaram valores superiores, em relação aos demais tratamentos, nos quais a taxa assimilatória líquida decresceu nitidamente . Diante disto, deve-se ressaltar que o bioestimulante aplicado via foliar à $333 \mathrm{ppm}$, contribuiu para aumentar a produção de matéria seca por unidade de área foliar no último intervalo de tempo. Tais resultados concordam com os observados por CASTRO (1981), quando verificou que a taxa de crescimento relativo mostrou-se mais elevada nas plantas tratadas com Agrostemin via foliar na concentração de 333 ppm, em relação ao controle.

Na TABELA 3A e Figura 3A, encontramse os valores médios obtidos para a taxa de crescimento relativo, considerando-se as plantas submetidas à aplicação de Agrostemin, tanto nas sementes como nas folhas. No intervalo (57-71), notou-se que este parâmetro foi maior nas plantas que receberam o tratamento $\mathrm{T}_{3}$, sendo que os demais tratamentos apresentaram valores próximos.

No intervalo seguinte (71-85), verifica-se que a taxa de crescimento relativo mostrou-se alta nas plantas que receberam o tratamento $T_{2}$, em relação as plantas tratadas tanto nas sementes quanto nas folhas. Dessa forma, cumpre ressaltar que o bioestimulante aplicado nas duas vias, semente $\mathrm{e}$ foliar, não promoveu incrementos neste parâmetro fisiológico, diminuindo-o. Portanto, pode-se sugerir que o Agrostemin empregado nestas condições, talvéz possa estar provocando um efeito inibitório, devido possivelmente a uma dosagem excessiva.

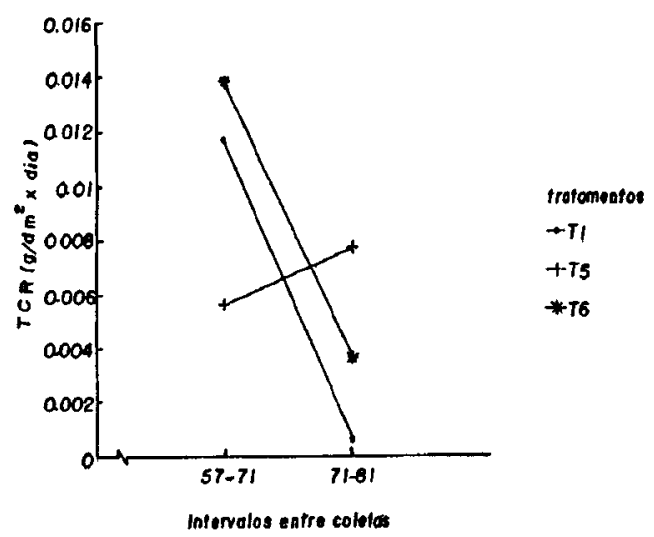

Figura 3: Valores médios obtidos para taxa de crescimento relativo, considerando-se as plantas que receberam Agrostemin, via foliar. 


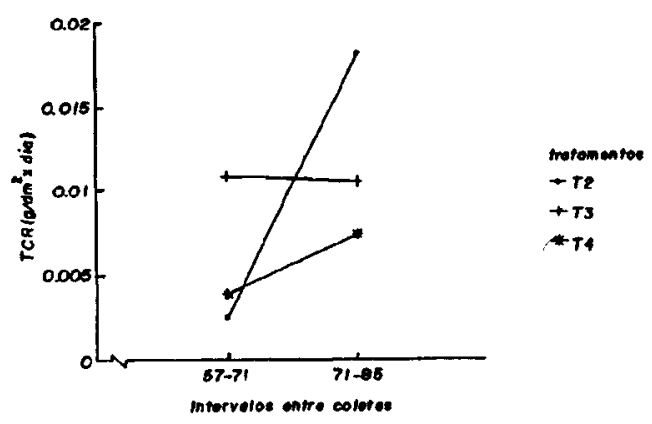

Figura 3A: Valores médios obtidos para taxa de crescimento relativo, considerando-se as plantas que receberam Agrostemin, tanto por via foliar como por semente.

\section{CONCLUSŐES}

A análise e a interpretação dos resultados obtidos, permitiram estabelecer as seguintes conclusões:
À medida que aumentou-se a concentração de Agrostemin de 333 ppm à 500 ppm, ocorreu uma diminuição na razão de área foliar. Não se observou aumento neste parâmetro, quando Agrostemin foi aplicado em dois estádios, via semente e via foliar.

Quando aplicou-se Agrostemin, via foliar, as plantas apresentaram redução na taxa assimilatória líquida, no intervalo 57-71; já no intervalo seguinte as plantas que receberam o tratamento $T_{\text {, apresenta- }}$ ram maiores valores para este parâmetro, ocorrendo o mesmo quando a aplicação foi em dois estádios na concentração de 333 ppm.

Agrostemin aplicado via semente e via foliar à $333 \mathrm{ppm}$, elevou a taxa de crescimento relativo no intervalo 57.71 , diminuindo no intervalo seguinte, quando a concentração aumentou de $333 \mathrm{ppm}$ a $500 \mathrm{ppm}$.

TABELA 3: Valores médios obtidos para a taxa de crescimento relativo, considerando-se os tratamentos $T_{1}$, $\mathrm{T}_{5}$ e $\mathrm{T}_{6}$, nos intervalos $57-71$ e $71-84$ dias pós-plantio, expressos $\mathrm{em} \mathrm{g/g.dia.}$

\begin{tabular}{lll}
\hline \multirow{2}{*}{ Tratamentos } & \multicolumn{2}{c}{ Intervalos } \\
\cline { 2 - 3 } $\mathrm{T}_{1}$ & $\frac{57-71}{0,0117}$ & $\mathbf{7 1 - 8 4}$ \\
$\mathrm{T}_{5}$ & 0,0056 & 0,0006 \\
$\mathrm{~T}_{6}$ & 0,0138 & 0,0077 \\
\hline \hline
\end{tabular}

TABELA 3A: Valores médios obtidos para a taxa de crescimento relativo, considerando-se os tratamentos $T_{2}$, $\mathrm{T}_{3}$ e $\mathrm{T}_{4}$, nos intervalos 57-71 e 71-85 dias pós-plantio, expressos em g/g.dia.

\begin{tabular}{lll}
\hline \hline Tratamentos & \multicolumn{2}{c}{ Intervalos } \\
\cline { 2 - 3 } $\mathrm{T}_{2}$ & $57-71$ & $71-84$ \\
$\mathrm{~T}_{3}$ & 0,0025 & 0,0182 \\
$\mathrm{~T}_{4}$ & 0,0108 & 0,0153 \\
\hline \hline
\end{tabular}

\section{REFERÊNCIAS BIBLIOGRÁFICAS}

BANZATTO, D.A; KRONKA, S.N. Experimentaç̃o agńcola. Jaboticabal: FUNEP, 1989. 247p.

BENINCASA, M.M.P. Análise de crescimento de plantas (noções básicas). Jaboticabal: FUNEP, 1988. 42p.

CASTRO, P.R.C. Análise de crescimento e produção de soja (Glycine max cv. Davis) sob efeito de fitorreguladores. Clência e Cultura, São Paulo, v.33, n.10, p.1346-1349, 1981 .

Sci. agric., Piracicaba, 52(2):339-345, mai./ago. 1995
CASTRO, P.R.C.; MORAES,R.S. Ação de fitorreguladores na produtividade da soja cultivar Davis. Anais da Escola. Superior de Agricultura "Luiz de Queiroz", Piracicaba, v.38, n.1, p.127-137, 1981.

CASTRO, P.R.C.; CONFORTO, E.; NICOLINI, E.M.; GABRIEL, J.L.C.; ISMAEL, J.J. Efeitos de reguladores e estimulantes vegetais no desenvolvimento do milho (Zea mays L. Cv. C-525). Anais da Escola Superoir de Agricultura "Luiz de Queiroz", Piracicaba, v.44, n.2, p.1079-1105, 1987. 
GAJIC, D.; VRBASKI, M. Identification of the effect of biorregulators from Agrostemma githago upon wheat in heterotrophic feeding with respect to Agrostemmin and Allantoin. Fragmenta Herbarium Croatica, v.7, p.1-6, 1972.

MAGALHÃES, AC.N. Análise quantitativa de crescimento. In: FERRI, M.G. Fisiologia vegetal. São Paulo: EPU/EDUSP, 1986. v.1, p.331-350.
RADFORD, P.J. Growth analysis formulae: their use and abuse. Crop Science, Madison, v.7, n.3, p.171-175, 1967.

STANKOVIC, D.; RAJKOVIC, N. Effect of Agrostemmin on pear. Acta Horticulturae, Wageningen, v.161, p.21l-278, 1984.

Recebido para publicação em 03.08 .94 Aceito para publicação em 25.06 .95 